\title{
METHOD FOR STEREO MAPPING BASED ON OBJECTARX AND PIPELINE TECHNOLOGY
}

\author{
Fengzhu Liu ${ }^{\text {a, } * \text {, Tianen Chen }}{ }^{\mathrm{a}}$, Zongjian Lin ${ }^{\mathrm{b}}$, Ying Yang ${ }^{\mathrm{a}}$ \\ ${ }^{\text {a }}$ Shandong University of Science and Technology, Qingdao, China, lucklfz@163.com.cn \\ ${ }^{\mathrm{b}}$ Chinese Academy of Surveying \& Mapping, Beijing, China, lincasm@163.casm.ac.cn
}

Commission I, ICWG I/V

KEY WORDS: Photogrammetry, Mapping, CAD, Plotter, Vector

\begin{abstract}
:
Stereo mapping is an important way to acquire 4D production. Based on the development of the stereo mapping and the characteristics of ObjectARX and pipeline technology, a new stereo mapping scheme which can realize the interaction between the AutoCAD and digital photogrammetry system is offered by ObjectARX and pipeline technology. An experiment is made in order to make sure the feasibility with the example of the software MAP-AT (Modern Aerial Photogrammetry Automatic Triangulation), the experimental results show that this scheme is feasible and it has very important meaning for the realization of the acquisition and edit integration.
\end{abstract}

\section{INTRODUCTION}

With the development of computer technology, digital image processing, pattern recognition, computer vision and other disciplines, development, digital photogrammetry's meaning far beyond the scope of traditional photogrammetry. Now it has entered digital photogrammetry stage.

In today's digital age, digital mapping products has more and more market share for its own advantage, all-digital stereo mapping has become the mainstream of production. Now in the domestic market, the all-digital photogrammetric systems which mainly used in the production of digital stereo mapping are JX4 digital photogrammetric system, VirtuoZo digital photogrammetric system and foreign SocetSet, SSK, LPS, etc. Especially domestic digital photogrammetric systems JX4 and VirtuoZo have significant contribution to technological progress and productional expansion of photogrammetry in our country. However, most of the stereo mapping software's function in graphics editing, graphic mapping aspects cannot satisfy demand. AutoCAD is the most widely used mapping system at home and abroad, its rich graphics capabilities, powerful editing capabilities, and a good user interface, since its inception by the majority of designers of all ages. Therefore, this paper propose an stereo mapping scheme with AutoCAD's vector graphics editor combined with AutoCAD and stereo mapping system closely, easy to implement the integration of collector and editor.

To address the communication between different systems, this paper presents the use of ObjectARX and pipeline communication technology to connect mapping system and AutoCAD system. ObjectARX is the second objectoriented development tools provided by AutoCAD, with good module, independent, high stability, and provides a powerful solution for users to develop applications based on AutoCAD platform. Pipe communication technology is common technology of inter-process communication, is an effective means to solve the connection between AutoCAD and mapping software that achieve their interaction and communication.

\section{IMPLEMENTED BASED ON OBJECTARX FUNCTION MODULE DESIGN}

\subsection{ObjectARX Introduction}

ObjectARX is not an independent development platform, but an object-oriented development environment and application program interface running on Visual $\mathrm{C}++$ and based on language $\mathrm{C}++$. It essence is a Windows dynamic link library(DLL), not only can contain executable code, but also to expand the use of library under a variety of resources. The advantages:

(1) Expand the application's features.

(2) Can be written in multiple languages, developers can choose the most appropriate language.

(3) Simplify the management of software projects.

(4) Help to save memory.

(5) Contribute to the sharing of resources.

(6) Help to localize an application.

(7) Help solve the platform differences.

As a Visual $\mathrm{C}++$ dynamic link library, ObjectARX has a big difference with other DLL which is strictly based on C++ language as a module of the Visual $\mathrm{C}++$ program. The ObjectARX provide its own grammar basis in $\mathrm{C}++$ language, it is a tool designed to the development of AutoCAD, and has a more targeted, is a strong secondary development interface provided by AutoCAD graphic system for users, allows the user to customize and extend the software functionality according to their special needs, making the mapping system has good flexibility.

\subsection{ObjectARX Programming Environment}

ObjectARX programming environment provides an objectoriented application programming interface, enabling developers to use, customize and extend AutoCAD, can easily use the development structure of AutoCAD, directly access to

\footnotetext{
* Fengzhu Liu, master, Shandong University of Science and Technology, China, lucklfz@ 163.com
} 
AutoCAD database structures, graphics systems, and custom local commands.

ObjectARX environment includes the following five classes and a library compatible with the original ADS libraries:

AcRx: provides a system-level classes and $\mathrm{C}++$ macros set for dynamic link library initialization, link, run-time of registration and, identification.

AcEd: editor class for registered local commands and system event notification

$\mathrm{AcDb}$ : provides class directly access to AutoCAD database structure, including physical and other graphic objects and layers, line types and other non-graphic objects.

AcGi: provides a graphical interface class for drawing AutoCAD entities.

AcGe: mainly used by AcDb classes, including 2D and 3D geometric objects related to the class.

Compatible with the global ADS library: This is a standard C library, from a functional perspective, which may include almost all the features of the five libraries above. ObjectARX library functions are:

Access to AutoCAD database;

Interaction with the AutoCAD editor;

Use development platform of software to create the user interface, and supports multiple document interface (MDI);

Creating user-defined class;

Build complex applications;

Interact with other programming environments.

\subsection{ObjectARX Application Preparation}

\section{ObjectARX application architecture}

ObjectARX application is a dynamic link library (DLL), AutoCAD through acrxEntryPoint() function call ARX application module. acrxEntryPoint() function is not only an entry point communicated ARX application with AutoCAD, but also ARX application asses the message to the application and returns a status code to a way of AutoCAD. acrxEntryPoint() function is specified as follows: extern"C"AcRx::AppRetCode

acrxEntryPoint(AcRx::AppMsgCode msg,void*pk)

\{

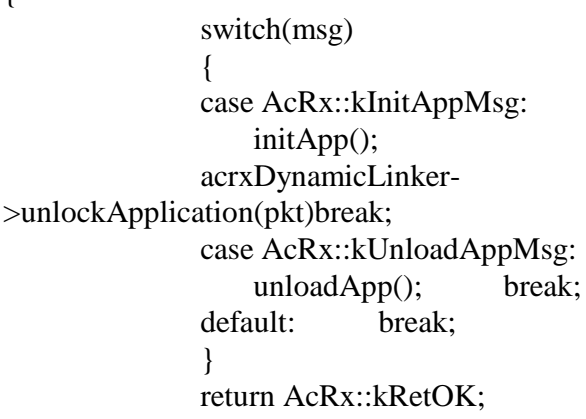

\}

To unload the ObjectARX application, you must unlock your application in acrxEntryPoint() function, the function code acrxDynamicLinker->unlockApplication(pkt) is to unlock the application, this statement is essential, otherwise, the application in AutoCAD will not be able to unload.

\section{Add the commend}

AutoCAD commands are stored in the command stack, defined by the AcEd CommandStack class, each command of
AutoCAD processes an instance in command stack. AutoCAD command stack contains internal commands and user-defined commands.

AcedRegCommangStack() macro provides function to operate command stack. AcEdCommandStack class contains a function of adding and removing command addCommand() and removeGroup(), which are explained below:

Acad::ErrorStatus AcEdCommandStack::

addCommand(const char*cmdGroupName,const char*

cmdGlobalName,const char*

cmdLocalName, Adesk::Int32

commandFlags, AcRxFunctionPtr functionAddr);

Virtual Acad::AcEdCommandStack::removeGroup(const char*GroupName);

ARX application can either define your own drawing commands, can also connect with other applications, is a powerful interface of AutoCAD mapping system. The following example shows the login and cancel of the order in which work() is a user-defined application command.

acedRegCmds->addCommand

("QDS_SPRING_CMD","WORK","WORK",ACRX_CMD_M

ODAL,\&work);

acedRegCmds->removeGroup("QDS_SPRING_CMD");

In the statement to use acedRegCmds-> addCommand() function to add the command, the command function sets void as a return value and cannot have the function parameters.

\section{Loading and unloading of ARX application}

In the main AutoCAD menu select Tools / Load Application, select to be load or unload ARX application through a dialog box ; or type ARX in AutoCAD command line, and then use the LOAD or UNLOAD to load or unload ARX application.

\section{Run the ARX application}

ARX application after loading, type local command, such as work, in the login group can execute applications.

Based on these features of ObjectARX, this paper achieve interaction with AutoCAD software platform, fully use of powerful editing features of AutoCAD, and communication between stereo mapping systems and other connections.

\section{PIPE COMMUNICATION TECHNOLOGY}

\subsection{Introduction to pipeline communication technology}

Pipe (Pipe) is an inter-process communication mechanism, a process writes data to the pipeline, and another process can read the data from the other end of the pipe. You can also transmit two-way information in the same pipe, and can achieve connection and interaction through the network with a process of the remote machine. Named pipes, with good flexibility, can conduct reliable communications between different processes on the same computer or between processes on different computers by network.

\subsection{Achievement of pipe line communication}

Each named pipe has a unique name to distinguish other named pipes in the system object-named list. First, the call CreateNamedPipe() function at an end of application that creates one or more of the named pipe instance and assign it a name, call CreateFile() or CallNamedPipe() function at the other end to connect to a named pipe instance whose name is 
known. For example, to achieve the process A and process B pipe communication between the need to complete the following tasks:

In the process A needs to do are:

(1) API function CreateNamedPipe create a named pipe instance.

(2) Use the API function ConnectNamedPipe to connect requests on a named pipe instance.

(3)Respectively, use the API function ReadFile and WriteFile to send and receive data.

(4) Use the API function DisconnectNamedPipe to close pipe connections, CloseHandle to close the instance handle.

In the process $B$ needs to do are:

(1) API function WaitNamePipe wait for a named pipe instance.

(2) Establish a connection using the API function CreateFile.

(3) Use WriteFile and ReadFile API function to send or receive data, respectively.

(4) using the API function CloseHandle to close the opened named pipe session.

This proposed solution requires to complete all work in process A on stereo mapping platform, complete all tasks in the process $\mathrm{B}$ on the AutoCAD platform. This allows an effective completion of the real-time, two-way data communication that is the basis for the integration of data collecting and editing.

\section{MAPPING PROGRAMME ACHIEVEMENT}

Chapters II and III of this article describes two techniques proposed in this paper, and you can be very clear understanding the specific ideas of the method of editing integrated mapping, that is, use ObjectARX to achieve the AutoCAD drawing operation, mapping software and AutoCAD will be connected using pipe technology, to achieve stereo mapping of the data collection, editing integration. Specific implementations as follows:

Start the data editing platform (AutoCAD) on the stereo mapping platform so that data collection and editing software to run at the same time, to ensure data editing and collection work simultaneously. Stereo mapping software as a collecting system to map, collecting vector data such as topography, surface feature; use ObjectARX and pipes to send the data obtained and other real-time interactive commands to AutoCAD's data set, according to interactive real-time command to update the AutoCAD drawing and the vector layer in mapping software to ensure data collected results and the edited results can synchronize updates on the two platforms. ObjectARX is a middleware of AutoCAD and mapping platform, and pipeline provides an effective transmission paths. System architecture can be roughly divided into the data collecting terminal (stereo mapping side), and data editing graphics terminal (AutoCAD drawing side).

Stereo mapping side mainly achieve stereoscopic 3D display and data collection to realize artificial three-dimensional data acquisition (or semi-automatic collection) in the threedimensional observation mode, data display overlay display and Manual interactive user interface, and will be three-dimensional vector data collected through the pipeline and ObjectARX send custom commands to the AutoCAD system for data editing and graphics mapping and follow-up.

In the AutoCAD side, mainly through the ObjectARX and the AutoCAD user interface to data acquisition and delivery of messages, to achieve real-time three-dimensional vector data display and editing, and editing through ObjectARX and pipe the results back to the mapping platform, interactive data acquisition and editing.

This allows the three-dimensional mapping data collection terminal to complete topography, surface features such as data collection, and the vector data will be written to the pipeline. Editing in AutoCAD platform, written by ARX application to read data pipeline, and the food intake data for editing, mapping, and ultimately get the vector line graph DLG. The general structure of the system shown in Figure:

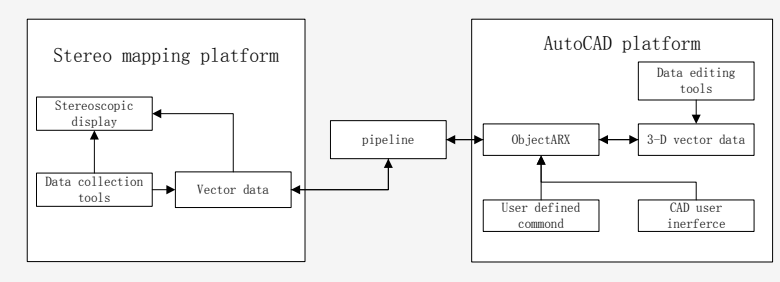

Figure 1. Mapping program structure design

\section{APPLICATION}

Modern air, aerial Automatic software MAP-AT is a fully digital photogrammetric system, simple operation, high automation, running fast, etc., can handle a variety of digital cameras and traditional film images scanned images, capable of stereo mapping data collection. In this paper, MAP-AT for the mapping platform, Visual $\mathrm{C}++$ for software development platform, AutoCAD2002 for data editing platform to Hainan digital Danzhou DLG production data for the experimental data in the experiment, the paper's editorial integrated mapping program design and implementation.

Overview of the surveyed area in Hainan: flying platform used by the Chinese Academy of Surveying and Mapping selfdeveloped unmanned aircraft, sensors for the Canon 5D mark2 single camera, the camera focal length of $35 \mathrm{~mm}$, flight altitude of 600 meters, the image resolution of $10 \mathrm{~cm}$, the flight time of 2010 July 30,1600 photo number, size of pieces relative to $5616 \times 3744$, laid an average of 5-6 baseline control points, the measured total area of 5 square kilometers.

Experiments on relatively simple hardware configuration requirements, a typical desktop computer or laptop can. Mapping can use the flash when the closed three dimensional optical mapping, and mapping using red and green glasses, either using normal mouse mapping, can also hand wheel / foot plate and three-dimensional mouse mapping.

Three-dimensional mapping mode can be hand wheel, mousedimensional images, or the mouse wheel to modify foot plate elevation for mapping. In the mapping process can be checked at any time in AutoCAD is the measured error vector (such as surface features cross, overlap, etc.), then you can start in the MAP-AT in AutoCAD, in the CAD interface to display the measured vector, as shown below. 


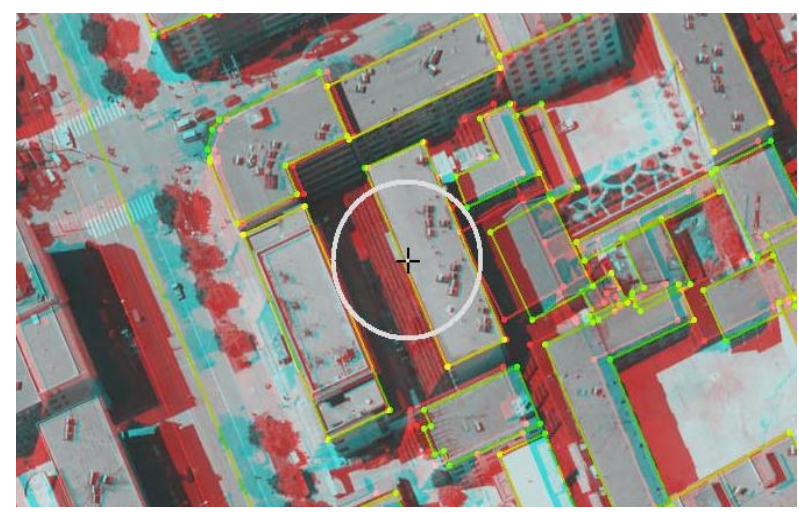

Figure 2. MapAT mapping platform renderings

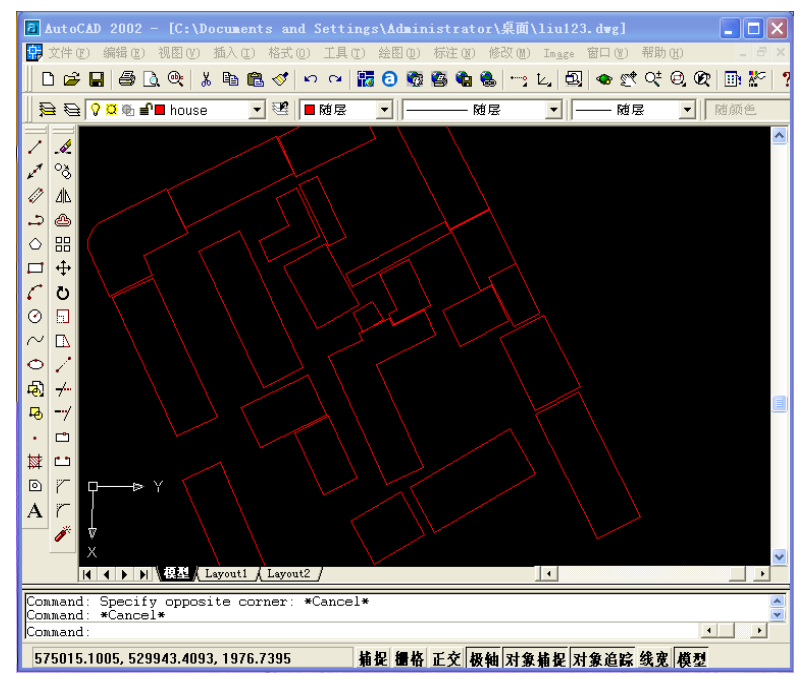

Figure 3. MappAT mapping output the results to AutoCAD

Figure 2 shows the MAP-AT digital photogrammetry system, acquisition of housing and other surface features, the results collected in AutoCAD editing platform is displayed (Figure 3), AutoCAD vector editing platform was completed for the nodes, line segments and surface, such as shift, zoom, rotate, connect, copy, disconnect, and recover operations.

Experimental results show that based on this technology ObjectARX and pipeline mapping method can easily achieve an integrated editing of vector data, directly in the AutoCAD platform for the collection of vector graphics to check, edit, has the following advantages:

(1) system, full use of AutoCAD software, powerful graphics editing features, making the mapping mapping and plotting efficiency is greatly improved;

(2) capture and edit simultaneously, not only makes data acquisition more intuitive and interactive approach makes the data more convenient data checking;

(3) ObjectARX and pipeline technology can be a different platform and AutoCAD mapping to connect different platforms to play their own advantages;

(4)In this paper, three-dimensional mapping program for data acquisition and editing software presents a new and effective design.

\section{CONCLUTION}

This proposed mapping method can make MAP-AT and other mapping software to effectively use the powerful editing capacity of AutoCAD, convenient, real-time for a variety of terrain, surface features such as vector data editing, mapping can be commonly encountered during the editing processing to speed up the efficiency of mapping, direct access to DLG, to meet the needs of users. This method is currently one of the initial attempt, in practical applications may also exist some problems, but also in practice to be perfect

\section{REFERENCE}

\section{References from Books:}

Zhang Zuxun, Zhang Jianqing, Digital Photogrammetry, Wuhan University Press.

Zhang Jianqing, Pan Li, Wang Shugen, photogrammetry: Wuhan University Press.

Ding Jinsheng, Chen Junyong, Li Deren, Liu Jingnan, Zhang Zuxun, Introduction to Geomatics: Wuhan University Press.

Li Shiguo, Advanced AutoCAD ARX programming and application development technology, Machinery industry press.

Dong Yude, Zhao Han, Secondary development technology of CAD, Hefei industrial university press.

\section{References from Journals:}

Zhang Zuxun, Zhang Jianqing, Zhang Li, Opportunities and Challenges for Development of Digital Photogrammetry, Journal of Wuhan Technical U niversity of Surveying and Mapping, 2000.

Zhou Zhonghai, Qu Huadong, Zhang Tao, Jiang Duwei, The VC++ naming pipeline the technology realizes, China Water Transport, 4(6). Ren Qingbo. Research and Implication on CAD and VC Data Interface Platform, Dalian University of Technology,2007.

Li Jianbo. Stereo Mapping using SSK Total Digital Photogrammetric System: Geomatics \& Spatial Innformation Technology, 31(5). 